\title{
A!
}

This is an electronic reprint of the original article.

This reprint may differ from the original in pagination and typographic detail.

Qin, Huajun; van Dijken, Sebastiaan

Nanometer-thick YIG-based magnonic crystals

Published in:

Applied Physics Letters

DOI:

$10.1063 / 5.0009807$

Published: 18/05/2020

Document Version

Publisher's PDF, also known as Version of record

Please cite the original version:

Qin, H., \& van Dijken, S. (2020). Nanometer-thick YIG-based magnonic crystals: Bandgap dependence on groove depth, lattice constant, and film thickness. Applied Physics Letters, 116(20), [202403].

https://doi.org/10.1063/5.0009807

This material is protected by copyright and other intellectual property rights, and duplication or sale of all or part of any of the repository collections is not permitted, except that material may be duplicated by you for your research use or educational purposes in electronic or print form. You must obtain permission for any other use. Electronic or print copies may not be offered, whether for sale or otherwise to anyone who is not an authorised user. 


\section{Nanometer-thick YIG-based magnonic crystals: Bandgap dependence on groove depth, lattice constant, and film thickness}

Cite as: Appl. Phys. Lett. 116, 202403 (2020); https://doi.org/10.1063/5.0009807

Submitted: 04 April 2020 . Accepted: 11 May 2020 . Published Online: 20 May 2020

Huajun Qin (D), and Sebastiaan van Dijken (i)

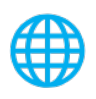

\section{ARTICLES YOU MAY BE INTERESTED IN}

Microwave assisted gating of spin wave propagation

Applied Physics Letters 116, 162403 (2020); https://doi.org/10.1063/5.0006945

Spin-orbit-torque magnonics

Journal of Applied Physics 127, 170901 (2020); https://doi.org/10.1063/5.0007095

Spinwave detection by nitrogen-vacancy centers in diamond as a function of probe-sample separation

Applied Physics Letters 116, 202401 (2020); https://doi.org/10.1063/1.5141921

\section{Lock-in Amplifiers up to $600 \mathrm{MHz}$}
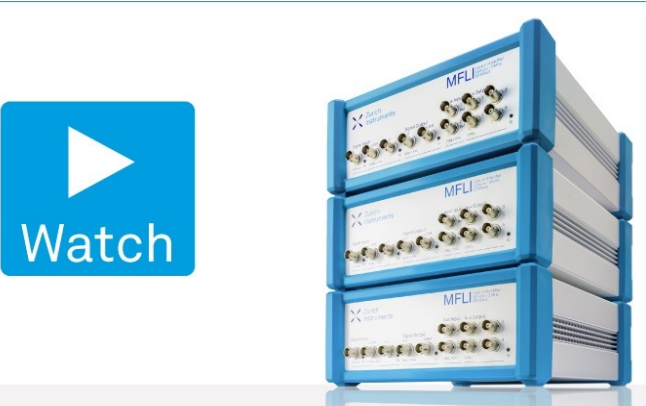


\title{
Nanometer-thick YIG-based magnonic crystals: Bandgap dependence on groove depth, lattice constant, and film thickness
}

Cite as: Appl. Phys. Lett. 116, 202403 (2020); doi: 10.1063/5.0009807

Submitted: 4 April 2020 - Accepted: 11 May 2020 .

Published Online: 20 May 2020

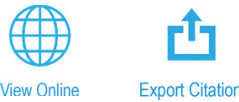

Huajun Qin $^{a)}$ (D) and Sebastiaan van Dijken ${ }^{\text {b) }}$ (iD

\author{
AFFILIATIONS \\ a) Author to whom correspondence should be addressed: huajun.qin@aalto.fi

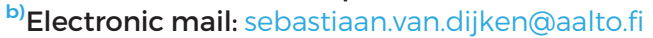

NanoSpin, Department of Applied Physics, Aalto University School of Science, P.O. Box 15100, Fl-00076 Aalto, Finland

\begin{abstract}
We report on bandgap tuning in magnonic crystals made of nanometer-thick yttrium iron garnet (YIG) films with CoFeB-filled grooves via a variation of the groove depth, lattice constant, and film thickness. Using broadband spin-wave spectroscopy, we demonstrate bandgap widening in a 260-nm-thick YIG crystal when the grooves are deepened from half to full film thickness. Importantly, low-loss spin-wave transmission in the allowed bands of the magnonic crystal is almost unaffected by the patterning of fully discrete YIG stripes. Downscaling of the YIG film thickness to $35 \mathrm{~nm}$ decreases the bandgap size through a flattening of the spin-wave dispersion relation. We show that a reduction in the lattice constant effectively compensates for this trend. Our experimental results are corroborated by micromagnetic simulations, providing relevant information for the design of ultrathin YIG-based magnonic crystals with optimized bandgaps and spin-wave transmission properties.
\end{abstract}

Published under license by AIP Publishing. https://doi.org/10.1063/5.0009807

Magnonics utilizing spin waves for information transport, storage, and processing offers an alternative for CMOS-based technology free of detrimental Joule heating at high operation frequency. ${ }^{1-4}$ The realization of magnonic devices requires active control of spin waves and low transmission losses. Magnonic crystals, i.e., metamaterials with periodically modulated magnetic properties, ${ }^{5-8}$ made of yttrium iron garnet (YIG) are promising candidates for performing such tasks because of ultralow magnetic damping. ${ }^{4,9}$ Thus far, several types of micrometer-thick YIG-based magnonic crystals have been explored. In YIG films with a periodic array of shallow grooves, ${ }^{10-14}$ for instance, bandgaps with sizes ranging from a few $\mathrm{MHz}$ to tens of $\mathrm{MHz}$ have been demonstrated. In this configuration, increasing the groove depth widens and deepens the bandgaps at the cost of reduced spin-wave transmission at allowed frequencies. ${ }^{11}$ Patterning of metallic stripes on top of a YIG film can open up similarly sized bandgaps while preserving low-loss transmission. ${ }^{15-18}$ However, inefficient Bragg scattering in such crystals limits the bandgap depth. Other micrometer-thick YIG-based magnonic crystals that have been explored include widthmodulated structures ${ }^{19}$ and 3D networks grown onto patterned GGG substrates. ${ }^{20}$ Programmable control of spin-wave transmission in micrometer-thick YIG films has been demonstrated using currentcarrying meander structures, ${ }^{21,22}$ optical absorbers, ${ }^{23}$ and strain coupling to a piezoelectric layer. ${ }^{24}$
Following advances in the growth of nanometer-thick YIG films with ultralow magnetic damping, ${ }^{25-31}$ the excitation and low-loss propagation of short-wavelength spin waves ${ }^{32-34}$ and downscaling of YIG-based magnonic crystals ${ }^{35}$ are now possible. Recently, we demonstrated the formation of bandgaps with sizes up to $200 \mathrm{MHz}$ in lattices comprising only a few discrete 260 -nm-thick YIG stripes. ${ }^{35}$ The YIG stripes in this study were separated by either air grooves or grooves that were filled with CoFeB. Compared to micrometer-thick YIG films, the opening of larger bandgaps with fewer lattice units in thin discrete YIG-based crystals is explained by stronger Bragg reflection on individual scatterers. Moreover, efficient coupling of thin YIG stripes via in-plane dipolar fields facilitates low-loss transmission in the allowed bands of the magnonic crystal. Here, we experimentally explore spinwave transmission in nanometer-thick YIG-based magnonic crystals further. Besides downscaling the YIG film thickness from $260 \mathrm{~nm}$ to $35 \mathrm{~nm}$, we also report on the effects of the groove depth and lattice constant.

We grew the YIG films on GGG(111) substrates using pulsed laser deposition (PLD). In the PLD chamber, the substrates were first degassed at $550^{\circ} \mathrm{C}$ for $15 \mathrm{~min}$. After this, the growth temperature $\left(800^{\circ} \mathrm{C}\right)$ and oxygen partial pressure $(0.13$ mbar $)$ were set and the films were deposited from a stoichiometric YIG target using an excimer laser with a pulse repetition rate of $2 \mathrm{~Hz}$ and a fluence of 
$1.8 \mathrm{~J} / \mathrm{cm}^{2}$. After film growth, the samples were annealed at $730^{\circ} \mathrm{C}$ for $10 \mathrm{~min}$ in 13 mbar oxygen before cooling to room temperature at a rate of $-3{ }^{\circ} \mathrm{C}$ per minute. The Gilbert damping parameter of the asgrown films was estimated as $(5 \pm 1.5) \times 10^{-4}$ using a vector network analyzer ferromagnetic resonance (VNA-FMR) technique. ${ }^{30}$ The YIG films were patterned into magnonic crystals by direct laser writing (DLW) lithography. As the first step, we exploited DLW to define an array of 1D stripes in a photoresist layer on top of YIG. Next, we used argon-ion milling to pattern four grooves of varying depths in the YIG films. In all samples, the grooves were filled with $\mathrm{Co}_{40} \mathrm{Fe}_{40} \mathrm{~B}_{20}(\mathrm{CoFeB})$ using magnetron sputtering. As a final step, the resist layer was lifted off by placing the samples in a bath of acetone. Transport across the magnonic crystals was characterized by broadband spin-wave spectroscopy. Transmission spectra were recorded by measuring the $S_{12}$ scattering parameter using a vector network analyzer. Two parallel microwave antennas with a width of $6 \mu \mathrm{m}$ excited and detected the propagating spin waves. The antennas consisted of $3 \mathrm{~nm} \mathrm{Ta} / 120 \mathrm{~nm}$ $\mathrm{Au}$ and were directly patterned on top of the YIG films using DLW lithography and lift-off. The distance between the two antennas was $200 \mu \mathrm{m}$ in all experiments.

Figures 1(a) and 1(b) show a schematic of the measurement geometry and a scanning electron microscopy (SEM) image of a magnonic crystal with four CoFeB-filled grooves. The lattice constant of the crystal is $a=50 \mu \mathrm{m}$, and the groove width is $w=5 \mu \mathrm{m}$. Hereafter, we refer to this magnonic crystal as a50w5. In broadband spin-wave spectroscopy measurements, a magnetic bias field is applied along the stripes to establish the Damon-Eshbach (DE) configuration. Figures 1 (c) and 1(f) present contour plots of spin-wave transmission spectra (amplitude of the $S_{12}$ scattering parameter) as a function of bias field for 260-nm-thick a50w5 crystals with a groove depth of $d=130 \mathrm{~nm}$ [Fig. 1(c)] and $260 \mathrm{~nm}$ [Fig. 1(f)]. The spin-wave transmission spectra of both crystals show multiple deep bandgaps. Within the gaps, efficient spin-wave rejection lowers the transmission signal to the measurement background level. In contrast, spin waves transmit efficiently between the two antennas in the allowed bands of the magnonic crystals. Weakening of the excitation efficiency gradually reduces the spinwave intensity with increasing frequency, which is a common effect for microwave antennas. We also observe a small difference in the spin-wave transmission signal for negative and positive bias fields [Figs. 1(c) and 1(f)]. This non-reciprocity is caused by dissimilar propagation of DE spin waves at the bottom and top surfaces of the YIG film $^{36,37}$ and the excitation configuration of the microwave antenna. ${ }^{38}$ The minimum frequency in Figs. 1(c) and 1(f) does not occur at zero magnetic field. The finite coercive field of the YIG film produces a small bias field offset. The direction of the field sweep during broadband spin-wave spectroscopy determines the polarity of this offset.

The measured bandgaps are formed by Bragg scattering of spin waves with wave vectors satisfying $k=n \pi / a$, where $n$ is the order number of the bandgap. ${ }^{5-8}$ For instance, the frequencies of the first six bandgaps in both a50w5 crystals [Figs. 1(d) and 1(g)] correspond to wave vectors $n \pi / a$, with $n=1-6$ and $a=50 \mu \mathrm{m}$ [Figs. 1(e) and 1(h)]. Here, the spin-wave dispersion relations are calculated using $f=\frac{\gamma \mu_{0}}{2 \pi} \sqrt{\left(H_{\text {ext }}+H_{\text {ani }}\right)\left(H_{\text {ext }}+H_{\text {ani }}+M_{\text {eff }}\right)+\frac{M_{\text {eff }}^{2}}{4}\left(1-e^{-2 k d}\right),{ }^{39}}$ with input parameters $\frac{\gamma}{2 \pi}=28 \mathrm{GHz} / \mathrm{T}, d=260 \mathrm{~nm}, \mu_{0} H_{\text {ext }}=5 \mathrm{mT}$, and magnetic anisotropy field $\mu_{0} H_{\text {ani }}=1.5 \mathrm{mT}$. The effective magnetization $\left(M_{\text {eff }}\right)$ of the $a 50 w 5$ crystal with 130 -nm-deep and 260-nm-deep grooves is $171 \mathrm{kA} / \mathrm{m}$ and $185 \mathrm{kA} / \mathrm{m}$, respectively. The values of $\mu_{0} H_{\mathrm{ani}}$ (a)

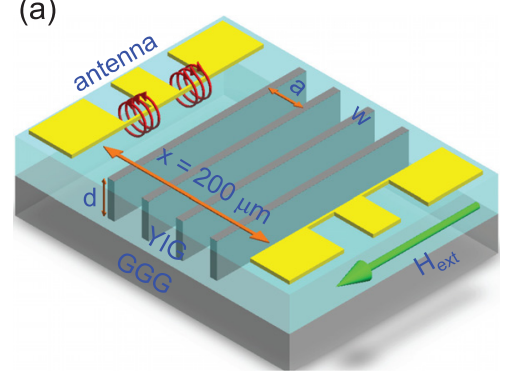

(b)

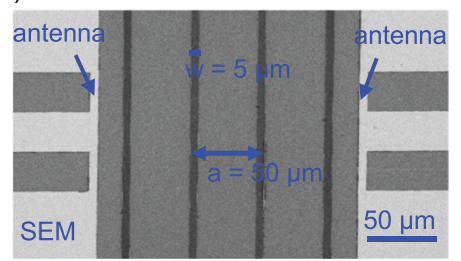

(c)

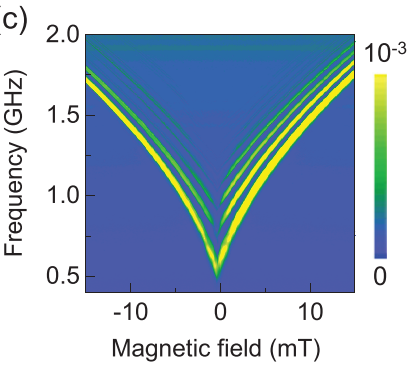

(f)

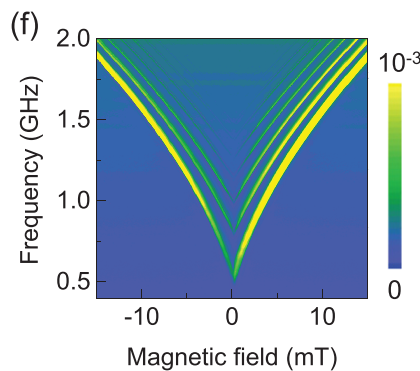

(d)

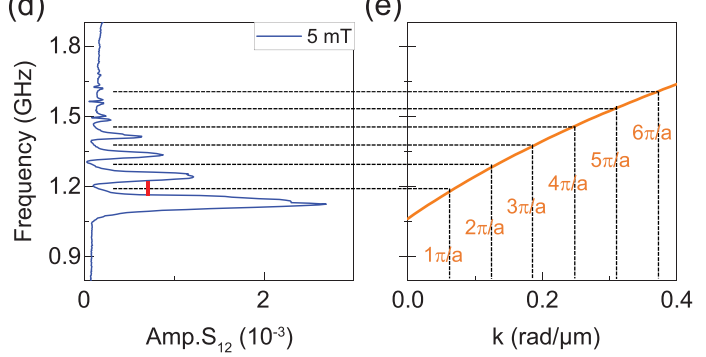

(g)

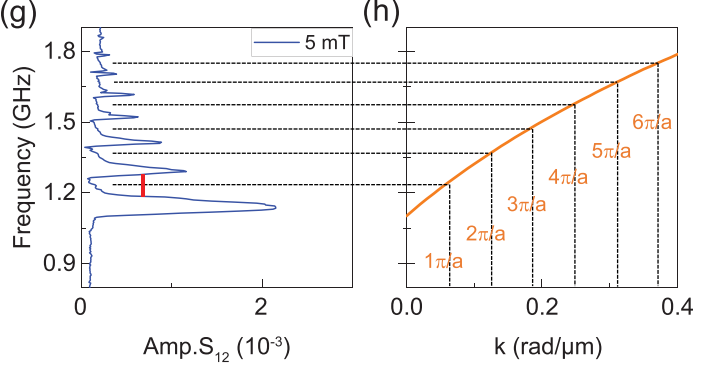

FIG. 1. (a) and (b) Schematic of the measurement geometry and SEM image of a a50w5 magnonic crystal consisting of four CoFeB-filled grooves. Two 6- $\mu$ m-wide microwave antennas are patterned on top of the YIG film. (c) Contour plot of spin-wave transmission spectra (amplitude of the $S_{12}$ scattering parameter) as a function of bias field for a a50w5 magnonic crystal with four 130-nm-deep CoFeB-filled grooves in a 260-nm-thick YIG film. (d) Spin-wave transmission spectrum of the same crystal for a bias field of $5 \mathrm{mT}$. (e) Dispersion relation of YIG for DE spin waves and a bias field of $5 \mathrm{mT}$. The vertical and horizontal dashed lines indicate the bandgap center frequencies and corresponding Bragg scattering wave vectors. (f)-(h) The same data as in (c)-(e) for a a50w5 magnonic crystal with four 260-nm-deep CoFeB-filled grooves in a 260-nm-thick YIG film. In (d) and $(g)$, the red line indicates the size of the first bandgap. 
and $M_{\text {eff }}$ are extracted from the dependence of the FMR frequency on the external bias field, which we obtained by recording the $S_{12}$ scattering parameter on both magnonic crystals [Figs. 1(c) and 1(f)]. A slight decrease in $M_{\text {eff }}$, compared to $188 \mathrm{kA} / \mathrm{m}$ extracted from VNA-FMR measurements on the as-grown YIG film, is caused most likely by argon-ion milling during fabrication. The larger value of $M_{\text {eff }}$ for the crystal with deeper grooves is attributed to a larger amount of $\mathrm{CoFeB}^{35}$ Since ion-milling-induced deterioration of YIG mainly occurs near the edges of the grooves, it has a minor effect on the spinwave transmission properties of YIG-based magnonic crystals with a micrometer-range lateral size.

We compare the $n=1$ bandgap center frequency and bandgap size of magnonic crystals with 130-nm-deep and 260-nm-deep grooves in Fig. 2. The size of the bandgap is measured at half the maximum signal of the second allowed transmission band, as indicated by the red lines in Figs. 1(d) and 1(g). The bandgap frequency follows the upshift of the YIG dispersion curve with increasing bias field. The small difference between the two curves in Fig. 2(a) is explained by a larger $M_{\text {eff }}$ in the crystal with 260-nm-deep CoFeB-filled grooves. The bandgap size of both crystals decreases with bias field [Fig. 2(b)]. This effect originates from a flattening of the spin-wave dispersion relation at large $H_{\text {ext. }}{ }^{30}$ The magnonic crystal with 260 -nm-deep grooves has a considerably larger bandgap. Widening of the bandgap in crystals with fully discrete YIG stripes reflects more efficient Bragg scattering compared to YIG films wherein the CoFeB-filled grooves extend to only half the film thickness. The formation of larger bandgaps usually comes at the cost of higher transmission losses in the allowed bands of a magnonic crystal. In our hybrid lattices, $\mathrm{CoFeB}$ limits spin-wave losses by enhancing the dynamic dipolar coupling field between discrete YIG stripes. ${ }^{35}$ The nearly identical transmission intensities measured on crystals with 130-nm-deep and 260-nm-deep CoFeB-filled grooves confirm this effect [Figs. 1(d) and 1(g)]. Similar dependencies on the magnetic bias field and groove depth as shown in Fig. 2 are also obtained for the $n=2-6$ bandgaps.

We now discuss the evolution of bandgap parameters with reducing film thickness and lattice constant. To gauge the thickness dependence, we fabricated a20w2.5 magnonic crystals with $d=260 \mathrm{~nm}$, $225 \mathrm{~nm}, 163 \mathrm{~nm}, 135 \mathrm{~nm}, 65 \mathrm{~nm}$, and $35 \mathrm{~nm}$. The four CoFeB-filled grooves extended fully to the GGG substrate in all crystals. Figures 3(a) and 3(d) show contour plots of spin-wave transmission spectra (amplitude of the $S_{12}$ scattering parameter) as a function of bias field for the 260-nm-thick and 65-nm-thick crystals. At least two bandgaps corresponding to the $n=1$ and $n=2$ Bragg conditions are observed
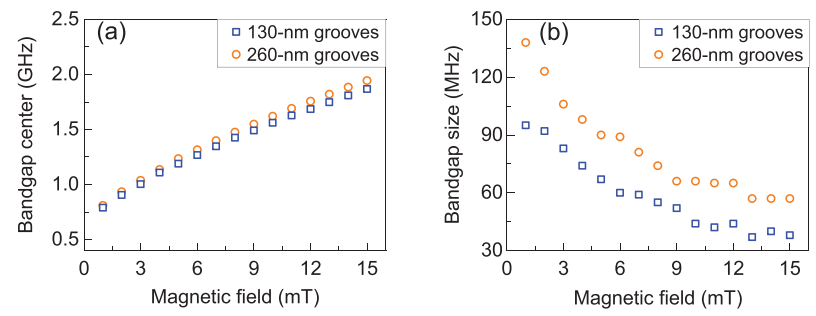

FIG. 2. (a) and (b) Bandgap center frequency and bandgap size as a function of magnetic bias field for $n=1$. The data are extracted from spin-wave transmission spectra recorded on 260-nm-thick a50w5 magnonic crystals with four 130-nm-deep or 260-nm-deep CoFeB-filled grooves.
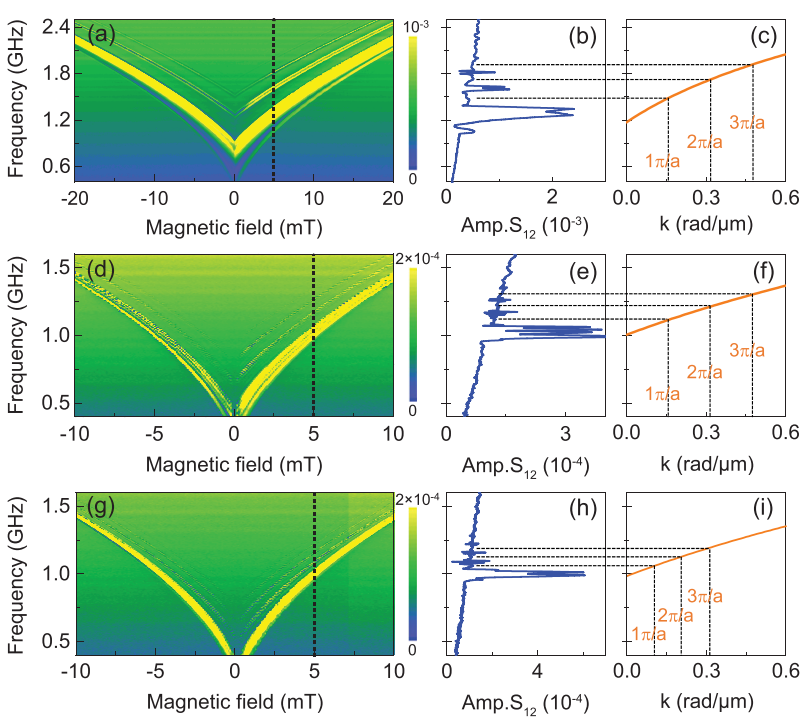

FIG. 3. (a) Contour plot of spin-wave transmission spectra (amplitude of the $S_{12}$ scattering parameter) as a function of bias field for a a20W2.5 magnonic crystal with four 260-nm-deep CoFeB-filled grooves in a 260-nm-thick YIG film. (b) Spin-wave transmission spectrum of the same crystal for a bias field of $5 \mathrm{mT}$. (c) Dispersion relation of YIG for DE spin waves and a bias field of $5 \mathrm{mT}$. The vertical and horizontal dashed lines indicate the bandgap center frequencies and corresponding Bragg scattering wave vectors. (d)-(f) The same data as in (a)-(c) for a a20w2.5 magnonic crystal with four 65-nm-deep CoFeB-filled grooves in a 65-nm-thick YIG film. (g)-(i) The same data as in (d)-(f) for $a=30 \mu \mathrm{m}$ instead of $a=20 \mu \mathrm{m}$.

in both measurements [Figs. 3(b) and 3(c) and 3(e) and 3(f)]. Compared to the 260-nm-thick magnonic crystal, the bandgap frequency and bandgap size are smaller for the 65-nm-thick crystal. Moreover, the spin-wave signal in the allowed bands is weaker. A lowering of the spin-wave decay length with reducing YIG film thickness explains the latter effect. ${ }^{30}$ To test the influence of the lattice constant, we fabricated 65-nm-thick magnonic crystals with $a=40 \mu \mathrm{m}$, $30 \mu \mathrm{m}, 20 \mu \mathrm{m}$, and $15 \mu \mathrm{m}$. Figures 3(g)-3(i) show experimental data for a lattice constant of $30 \mu \mathrm{m}$. Compared to $a=20 \mu \mathrm{m}$ as shown in Figures $3(\mathrm{~d})-3(\mathrm{f})$, the bandgap frequency and bandgap size are smaller for $a=30 \mu \mathrm{m}$.

To gain more insight into spin-wave transmission and bandgap formation in our nanometer-thick YIG-based magnonic crystals, we performed micromagnetic simulations in Mumax $3 .{ }^{40}$ We considered a $655-\mu$ m-long YIG structure with CoFeB-filled grooves separating fully discrete YIG stripes. The simulation area was discretized into $16384 \times 2 \times 4$ cells. One-dimensional periodic boundary conditions were applied along the $y$-axis to mimic an infinitely long crystal along this direction. To reduce spin-wave reflections from the edges of the simulation area, we added two 5 - $\mu$ m-wide regions with a Gilbert damping parameter of 0.5 . As input parameters, we used $M_{\mathrm{s}}=185 \mathrm{kA} / \mathrm{m}$ (YIG), $\quad M_{\mathrm{s}}=1150 \mathrm{kA} / \mathrm{m} \quad(\mathrm{CoFeB}), \quad A_{\mathrm{ex}}=3.1 \mathrm{pJ} / \mathrm{m} \quad(\mathrm{YIG}), \quad$ and $A_{\mathrm{ex}}=16 \mathrm{pJ} / \mathrm{m}(\mathrm{CoFeB})$. The damping parameters of YIG and CoFeB were set to 0.002 and 0.005 , respectively. We included a $0.16-\mu \mathrm{m}$-wide non-magnetic layer between the YIG stripes and $\mathrm{CoFeB}$ to account for ion-beam-induced changes of YIG near the milling edges of our experimental samples. ${ }^{35}$ Spin waves were excited at $x=0$ using a $0.1 \mathrm{mT}$ sincfunction-type magnetic field pulse. A bias field along $y$ established the 
DE geometry. After excitation, the time evolution of the $x$-component of magnetization $\left(m_{x}\right)$ was recorded for $200 \mathrm{~ns}$. Spatially resolved spinwave intensity maps were obtained by Fourier-transforming the evolution of $m_{x}$ on a cell-by-cell basis.

Figures 4(a) and 4(c) show the simulated spatial distribution of spin-wave intensity in $a 20 w 2.5$ magnonic crystals with thicknesses of $260 \mathrm{~nm}$ and $65 \mathrm{~nm}$, respectively. The bias field in the simulations is $5 \mathrm{mT}$. The line profiles in Figs. 4(b) and 4(d) are taken at $x=200 \mu \mathrm{m}$, mimicking the separation of microwave antennas in our experiments. The simulated spin-wave transmission spectra reproduce the main experimental findings [Figs. 3(b) and 3(e)], including the formation of bandgaps if the wave vector matches a Bragg condition, the narrowing of bandgaps with decreasing film thickness, and a reduction of the transmission signal in the 65-nm-thick magnonic crystal. The measured decrease in the bandgap size with increasing lattice constant [Figs. 3(g) and $3(\mathrm{~h})$ ] is also replicated by the micromagnetic simulations [Figs. 4(e) and 4(f)]. The spatial maps of Figs. 4(a), 4(c), and 4(e) show nodes within the allowed bands of the magnonic crystal, demonstrating partial confinement of spin waves within the discrete YIG stripes.

Figure 5 summarizes how the measured (solid symbols) and simulated (open symbols) center frequency and size of the $n=1$ bandgap vary with the crystal thickness and lattice constant in a magnetic bias field of $5 \mathrm{mT}$. A reduction in the YIG film thickness from $260 \mathrm{~nm}$ to $35 \mathrm{~nm}$ shifts the bandgap frequency down from $1.47 \mathrm{GHz}$ to $1.05 \mathrm{GHz}$ [Fig. 5(a)], and it decreases the bandgap size from $200 \mathrm{MHz}$ to just $40 \mathrm{MHz}$ [Fig. 5(b)]. Both scaling effects are caused by a flattening of
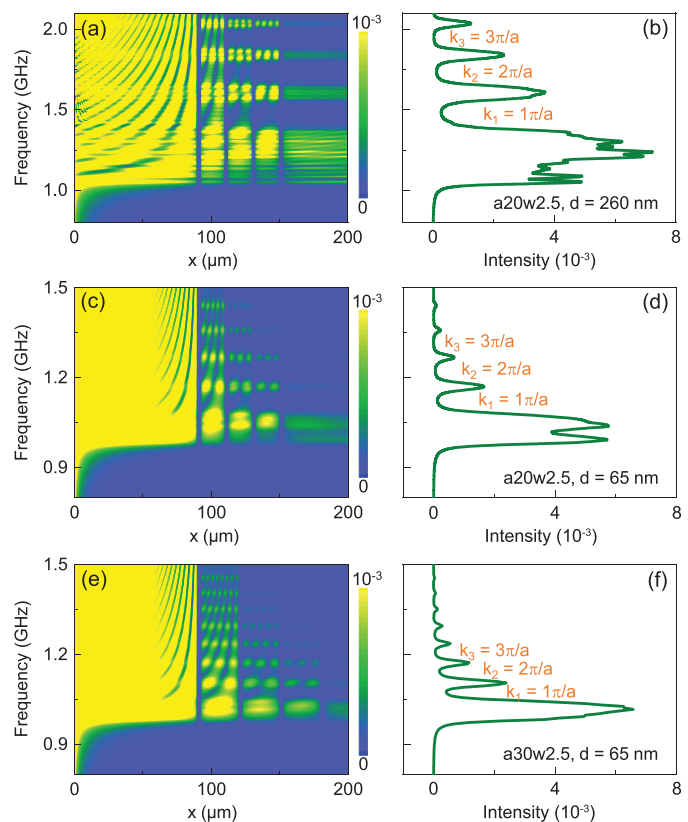

FIG. 4. (a) and (b) Simulated spatial distribution of spin-wave intensity and corresponding line profiles taken at $x=200 \mu \mathrm{m}$ for a a20w2.5 magnonic crystal with four CoFeB-filled grooves and a thickness of $260 \mathrm{~nm}$. (c) and (d) The same data as in $(a, b)$ for a a20w2.5 magnonic crystal with four CoFeB-filled grooves and a thickness of $65 \mathrm{~nm}$. (e) and (f) The same data as in (c) and (d) for $a=30 \mu \mathrm{m}$ instead of $a=20 \mu \mathrm{m}$. The grooves extend over the full thickness of the YIG film in all simulations. The magnetic bias field is $5 \mathrm{mT}$.
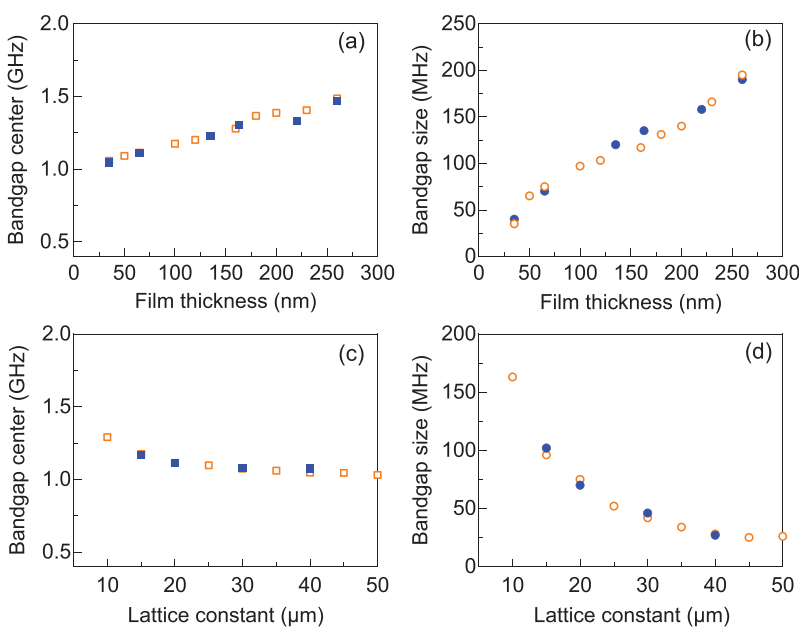

FIG. 5. (a) and (b) Center frequency and size of the $n=1$ bandgap as a function of YIG film thickness for a20w2.5 crystals with four CoFeB-filled grooves. (c) and (d) Center frequency and size of the $n=1$ bandgap as a function of lattice constant for $65-n m$-thick YIG films with four CoFeB-filled grooves. The data represented by solid and open symbols are extracted from experiments and simulations, respectively. The magnetic bias field is $5 \mathrm{mT}$.

the spin-wave dispersion curve, as illustrated for $d=260 \mathrm{~nm}$ and $d=65 \mathrm{~nm}$ in Figs. 3(c) and 3(f). The evolution of bandgap parameters with the lattice constant is depicted in Figs. 5(c) and 5(d) for magnonic crystals with a groove width of $2.5 \mu \mathrm{m}$, a thickness of $65 \mathrm{~nm}$, and four 65-nm-deep CoFeB-filled grooves. Reducing the lattice constant from $50 \mu \mathrm{m}$ to $10 \mu \mathrm{m}$ increases the bandgap frequency only slightly from $1.03 \mathrm{GHz}$ to $1.29 \mathrm{GHz}$ because the spin-wave dispersion curve of the 65-nm-thick YIG film is relatively flat [Fig. 5(c)]. The growing frequency gap between the $n=1$ and $n=2$ Bragg scattering conditions with decreasing lattice constant [compare Figs. 4(d) and 4(f)], however, enlarges the $n=1$ bandgap size significantly from $26 \mathrm{MHz}$ to $163 \mathrm{MHz}$ [Fig. 5(d)].

In conclusion, we investigated spin-wave transmission in nanometer-thick YIG-based magnonic crystals with CoFeB-filled grooves using broadband spin-wave spectroscopy and micromagnetic simulations. Effects of the groove depth, film thickness, and lattice constant on magnonic bandgaps and transmission losses were systematically gauged. We find that a change from half-deep grooves in a $260-\mathrm{nm}$ thick YIG film to fully discrete YIG stripes does enlarge the bandgaps while maintaining low-loss transmission at allowed frequencies. Downscaling of the YIG film thickness decreases the bandgap size, but this effect is offset by a concurrent reduction in the lattice constant. For $65-\mathrm{nm}$-thick YIG-based crystals, we find that $100-200 \mathrm{MHz}$ bandgaps are feasible. We point out that the use of smaller lattice constants allows for a reduction of the antenna separation, a scaling effect that could be exploited to compensate for higher transmission losses in ultrathin YIG films.

This work was supported by the Academy of Finland (Grant Nos. 317918, 316857, and 325480). Lithography was performed at the Micronova Nanofabrication Center, supported by Aalto University. We also acknowledge the computational resources provided by the Aalto Science-IT project. 


\section{DATA AVAILABILITY}

The data that support the findings of this study are available from the corresponding authors upon reasonable request.

\section{REFERENCES}

${ }^{1}$ A. Khitun, M. Bao, and K. L. Wang, "Magnonic logic circuits," J. Phys. D: Appl. Phys. 43, 264005 (2010).

${ }^{2}$ A. V. Chumak, V. I. Vasyuchka, A. A. Serga, and B. Hillebrands, "Magnon spintronics," Nat. Phys. 11, 453-461 (2015).

${ }^{3}$ S. A. Nikitov, D. V. Kalyabin, I. V. Lisenkov, A. Slavin, Y. N. Barabanenkov, S. A. Osokin, A. V. Sadovnikov, E. N. Beginin, M. A. Morozova, Y. A. Filimonov, Y. V. Khivintsev, S. L. Vysotsky, V. K. Sakharov, and E. S. Pavlov, "Magnonics: A new research area in spintronics and spin wave electronics," Phys.-Usp. 58, 1002 (2015).

${ }^{4}$ A. V. Chumak, A. A. Serga, and B. Hillebrands, "Magnonic crystals for data processing," J. Phys. D: Appl. Phys. 50, 244001 (2017).

${ }^{5}$ G. Gubbiotti, S. Tacchi, M. Madami, G. Carlotti, A. O. Adeyeye, and M. Kostylev, "Brillouin light scattering studies of planar metallic magnonic crystals," J. Phys. D: Appl. Phys. 43, 264003 (2010).

${ }^{6}$ V. V. Kruglyak, S. O. Demokritov, and D. Grundler, "Magnonics," J. Phys. D: Appl. Phys. 43, 264001 (2010).

${ }^{7}$ B. Lenk, H. Ulrichs, F. Garbs, and M. Mnzenberg, "The building blocks of magnonics," Phys. Rep. 507, 107- 136 (2011).

${ }^{8} \mathrm{M}$. Krawczyk and D. Grundler, "Review and prospects of magnonic crystals and devices with reprogrammable band structure," J. Phys.: Condens. Matter 26, 123202 (2014).

${ }^{9}$ A. A. Serga, A. V. Chumak, and B. Hillebrands, "YIG magnonics," J. Phys. D: Appl. Phys. 43, 264002 (2010).

${ }^{10}$ A. V. Chumak, A. A. Serga, B. Hillebrands, and M. P. Kostylev, "Scattering of backward spin waves in a one-dimensional magnonic crystal," Appl. Phys. Lett. 93, 022508 (2008)

${ }^{1}$ A. V. Chumak, A. A. Serga, S. Wolff, B. Hillebrands, and M. P. Kostylev, "Scattering of surface and volume spin waves in a magnonic crystal," Appl. Phys. Lett. 94, 172511 (2009).

${ }^{12}$ E. N. Beginin, Y. A. Filimonov, E. S. Pavlov, S. L. Vysotskii, and S. A. Nikitov, "Bragg resonances of magnetostatic surface spin waves in a layered structure: Magnonic crystal-dielectric-metal,” Appl. Phys. Lett. 100, 252412 (2012).

${ }^{13}$ C. L. Ordóñez-Romero, Z. Lazcano-Ortiz, A. Drozdovskii, B. Kalinikos, M Aguilar-Huerta, J. L. Domínguez-Juárez, G. Lopez-Maldonado, N. Qureshi, O. Kolokoltsev, and G. Monsivais, "Mapping of spin wave propagation in a onedimensional magnonic crystal,” J. Appl. Phys. 120, 043901 (2016).

${ }^{14}$ A. V. Sadovnikov, V. A. Gubanov, S. E. Sheshukova, Y. P. Sharaevskii, and S. A. Nikitov, "Spin-wave drop filter based on asymmetric side-coupled magnonic crystals," Phys. Rev. Appl. 9, 051002 (2018).

${ }^{15}$ M. Inoue, A. Baryshev, H. Takagi, P. B. Lim, K. Hatafuku, J. Noda, and K. Togo, "Investigating the use of magnonic crystals as extremely sensitive magnetic field sensors at room temperature," Appl. Phys. Lett. 98, 132511 (2011).

${ }^{16}$ M. Mruczkiewicz, E. S. Pavlov, S. L. Vysotskii, M. Krawczyk, Y. A. Filimonov, and S. A. Nikitov, "Magnonic bandgaps in metalized 1D YIG magnonic crystals,” IEEE Trans. Magn. 50, 1-3 (2014).

${ }^{17}$ V. D. Bessonov, M. Mruczkiewicz, R. Gieniusz, U. Guzowska, A. Maziewski, A. I. Stognij, and M. Krawczyk, "Magnonic band gaps in YIG-based one-dimensional magnonic crystals: An array of grooves versus an array of metallic stripes," Phys. Rev. B 91, 104421 (2015).

${ }^{18}$ T. Goto, K. Shimada, Y. Nakamura, H. Uchida, and M. Inoue, "One-dimensional magnonic crystal with $\mathrm{Cu}$ stripes for forward volume spin waves," Phys. Rev. Appl. 11, 014033 (2019).

${ }^{19}$ A. A. Nikitin, A. B. Ustinov, A. A. Semenov, A. V. Chumak, A. A. Serga, V. I. Vasyuchka, E. Lähderanta, B. A. Kalinikos, and B. Hillebrands, "A spin-wave logic gate based on a width-modulated dynamic magnonic crystal," Appl. Phys. Lett. 106, 102405 (2015).

${ }^{20}$ E. N. Beginin, A. V. Sadovnikov, A. Y. Sharaevskaya, A. I. Stognij, and S. A. Nikitov, "Spin wave steering in three-dimensional magnonic networks," Appl. Phys. Lett. 112, 122404 (2018).
${ }^{21}$ A. V. Chumak, T. Neumann, A. A. Serga, B. Hillebrands, and M. P. Kostylev, "A current-controlled, dynamic magnonic crystal," J. Phys. D: Appl. Phys. 42, 205005 (2009).

${ }^{22}$ A. V. Chumak, V. S. Tiberkevich, A. D. Karenowska, A. A. Serga, J. F. Gregg, A. N. Slavin, and B. Hillebrands, "All-linear time reversal by a dynamic artificial crystal,” Nat. Commun. 1, 141 (2010).

${ }^{23}$ M. Vogel, A. V. Chumak, E. H. Waller, T. Langner, V. I. Vasyuchka, B. Hillebrands, and G. von Freymann, "Optically reconfigurable magnetic materials," Nat. Phys. 11, 487 (2015).

${ }^{24}$ A. V. Sadovnikov, A. A. Grachev, S. E. Sheshukova, Y. P. Sharaevskii, A. A. Serdobintsev, D. M. Mitin, and S. A. Nikitov, "Magnon straintronics: Reconfigurable spin-wave routing in strain-controlled bilateral magnetic stripes," Phys. Rev. Lett. 120, 257203 (2018).

${ }^{25}$ B. Heinrich, C. Burrowes, E. Montoya, B. Kardasz, E. Girt, Y.-Y. Song, Y. Sun, and $\mathrm{M}$. Wu, "Spin pumping at the magnetic insulator (YIG)/normal metal (Au) interfaces," Phys. Rev. Lett. 107, 066604 (2011).

${ }^{26}$ Y. Sun, Y.-Y. Song, H. Chang, M. Kabatek, M. Jantz, W. Schneider, M. Wu, H. Schultheiss, and A. Hoffmann, "Growth and ferromagnetic resonance properties of nanometer-thick yttrium iron garnet films," Appl. Phys. Lett. 101, 152405 (2012).

${ }^{27}$ C. Hahn, G. de Loubens, O. Klein, M. Viret, V. V. Naletov, and J. Ben Youssef, "Comparative measurements of inverse spin Hall effects and magnetoresistance in YIG/Pt and YIG/Ta," Phys. Rev. B 87, 174417 (2013).

${ }^{28}$ H. Chang, P. Li, W. Zhang, T. Liu, A. Hoffmann, L. Deng, and M. Wu, "Nanometer-thick yttrium iron garnet films with extremely low damping," IEEE Magn. Lett. 5, 1-4 (2014).

${ }^{29}$ H. Yu, O. d'Allivy Kelly, V. Cros, R. Bernard, P. Bortolotti, A. Anane, F. Brandl, R. Huber, I. Stasinopoulos, and D. Grundler, "Magnetic thin-film insulator with ultra-low spin wave damping for coherent nanomagnonics," Sci. Rep. 4, 6848 (2015)

${ }^{30}$ H. Qin, S. J. Hämäläinen, K. Arjas, J. Witteveen, and S. van Dijken, "Propagating spin waves in nanometer-thick yttrium iron garnet films: Dependence on wave vector, magnetic field strength, and angle," Phys. Rev. B 98, 224422 (2018).

${ }^{31}$ L. Soumah, N. Beaulieu, L. Qassym, C. Carrétéro, E. Jacquet, R. Lebourgeois, J. Ben Youssef, P. Bortolotti, V. Cros, and A. Anane, "Ultra-low damping insulating magnetic thin films get perpendicular," Nat. Commun. 9, 3355 (2018).

${ }^{32}$ H. Yu, O. d' Allivy Kelly, V. Cros, R. Bernard, P. Bortolotti, A. Anane, F, Brandl, F. Heimbach, and D. Grundler, "Approaching soft x-ray wavelengths in nanomagnet-based microwave technology,” Nat. Commun. 7, 11255 (2016).

${ }^{33}$ C. Liu, J. Chen, T. Liu, F. Heimbach, H. Yu, Y. Xiao, J. Hu, M. Liu, H. Chang, T. Stueckler, S. Tu, Y. Zhang, Y. Zhang, P. Gao, Z. Liao, D. Yu, K. Xia, N. Lei, $\mathrm{W}$. Zhao, and $\mathrm{M}$. Wu, "Long-distance propagation of short-wavelength spin waves," Nat. Commun. 9, 738 (2018).

${ }^{34}$ J. Chen, T. Yu, C. Liu, T. Liu, M. Madami, K. Shen, J. Zhang, S. Tu, M. S. Alam, K. Xia, M. Wu, G. Gubbiotti, Y. M. Blanter, G. E. W. Bauer, and H. Yu, "Excitation of unidirectional exchange spin waves by a nanoscale magnetic grating," Phys. Rev. B 100, 104427 (2019).

${ }^{35}$ H. Qin, G.-J. Both, S. J. Hämäläinen, L. Yao, and S. van Dijken, "Low-loss YIGbased magnonic crystals with large tunable bandgaps," Nat. Commun. 9, 5445 (2018).

${ }^{36}$ T. Schneider, A. A. Serga, T. Neumann, B. Hillebrands, and M. P. Kostylev, "Phase reciprocity of spin-wave excitation by a microstrip antenna," Phys. Rev. B 77, 214411 (2008).

${ }^{37}$ K. L. Wong, L. Bi, M. Bao, Q. Wen, J. P. Chatelon, Y.-T. Lin, C. A. Ross, H. Zhang, and K. L. Wang, "Unidirectional propagation of magnetostatic surface spin waves at a magnetic film surface,” Appl. Phys. Lett. 105, 232403 (2014).

${ }^{38}$ K. Shibata, K. Kasahara, K. Nakayama, V. V. Kruglyak, M. M. Aziz, and T. Manago, "Dependence of non-reciprocity in spin wave excitation on antenna configuration," J. Appl. Phys. 124, 243901 (2018).

${ }^{39}$ B. A. Kalinikos and A. N. Slavin, "Theory of dipole-exchange spin wave spectrum for ferromagnetic films with mixed exchange boundary conditions," J. Phys. C 19, 7013 (1986)

${ }^{40}$ A. Vansteenkiste, J. Leliaert, M. Dvornik, M. Helsen, F. Garcia-Sanchez, and B. Van Waeyenberge, "The design and verification of MuMax3," AIP Adv. 4, 107133 (2014). 